\title{
Moving window generator for reading experiments
}

\author{
NAOYUKI OSAKA \\ Kyoto University, Kyoto, Japan \\ and \\ KOICHI ODA \\ Tokyo Woman's Christian University, Tokyo, Japan
}

\begin{abstract}
An inexpensive PC-based moving window generator with an eye movement recording system is described. The moving window technique, activated by current eye movements, has an advantage over the fixed window technique in measuring the effective visual field size during reading. A variable rectangular window, through which the subject observes the text, is generated on a PC-controlled CRT screen. The system includes a frame buffer memory, an analog-to-digital conversion unit, and an eye movement recording system. The system works well for measuring approximate field size during reading.
\end{abstract}

It is known that the area of the visual field from which we can extract useful information during each eye fixation changes as task difficulty changes. This area, called the size of the effective visual field, is relatively large during visual search, but small during reading (Rayner \& Pollatsek, 1989). The size of the effective visual field during reading can be determined in a simple and primitive way with a tachistoscopic presentation technique in which a sentence or stimulus array is briefly flashed before the subject, who maintains a fixed eye position. The exposure time should be brief enough to preclude the influence of eye movements. The subject then reports what he/she observes either near the fixation point or at some distance from it (see, e.g., Feinberg, 1949; Marcel, 1974). This is known as a fixed-eye technique (Rayner \& Pollatsek, 1989). A primitive window technique, in which the subject is only able to see the text through a limited window, is more elaborate: The window size is changed systematically, so that the effective visual field can be measured. The window can be manipulated so that the eye is fixed but the text moves (Bouma \& de Voogd, 1974; Legge, Pelli, Rubin, \& Schleske, 1985; Newman, 1966), or so that the text is fixed while the eye moves (Poulton, 1962). In the former, a single line of a text moves from right to left on the screen; in the latter, the aperture passes over the fixed text at a constant speed (Rayner \& Pollatsek, 1989). It should be noted that eye movements are not used to control the moving window in such studies.

This work was supported in part by Grants in Aid 01510061, 03401003,03801010 , and 03551002 to N.O. from the Ministry of Education, Japan. Thanks are due Hitoshi Tsuji and Hiroshi Ashida for their help in computer programming. Requests for reprints should be sent to N. Osaka, Department of Psychology, Faculty of Letters, Kyoto University, Kyoto 606, Japan (e-mail: b52046@tansei.cc.u-tokyo.ac.jp).
A more sophisticated and natural way to measure the effective visual field size is the moving-window technique introduced by McConkie and Rayner (1975) in which the current eye position activates the movement of the current window in which text is presented.

Such a technique relies on sophisticated eye movement equipment interfaced with a high-speed computer system, connected to a CRT. McConkie and Rayner (1975) used an expensive computer system (Digital Equipment Corporation's PDP-6) coupled with an eye movement recorder (hereafter EMR; Biometrics, Model SG). Rayner, Well, Pollatsek, and Bertera (1982) used the expensive Stanford Research Institute Dual Purkinje eyetracker, interfaced to a high-speed Hewlett-Packard 2100 computer, to conduct their moving window experiments. The high cost of such equipment limits the use of moving window studies. In the present report, we introduce a relatively inexpensive alternative.

\section{OVERVIEW OF THE SYSTEM}

We have developed an inexpensive PC-based system with an inexpensive frame buffer and fast analog-to-digital converter, which should enable the wide use of the moving window procedure in reading. A block diagram of the system is shown in Figure 1. A frame buffer with $768 \mathrm{~K}$ of RAM was used as the video memory. This RAM area is independent of the built-in video RAM, so it can be superimposed on the video RAM that contains text. An eye movement recorder and data processing unit are also required for generating the moving window (see Young \& Sheena, 1975, and McConkie, Zola, Wolverton, \& Burns, 1978 , for eye movement measuring systems presented in detail). Although the moving window generator introduced here works on a frame-by-frame basis $(33 \mathrm{msec})$, it appears to be sufficient for measuring approximate effec- 


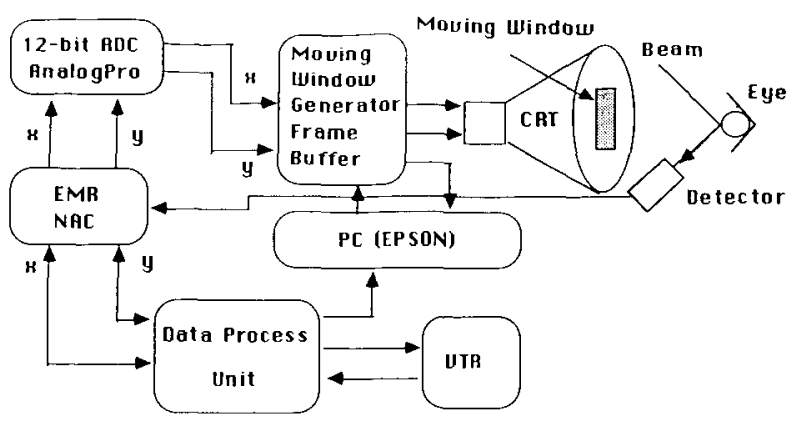

Figure 1. Block diagram of a PC-based moving window generator. EMR and VTR designate eye movement recorder and videotape recorder, respectively.

tive visual field sizes during reading. The time lag between eye movement and window movement (in the present study) is not as short as that of the SRI eyetracker interfaced with the Hewlett-Packard 2100 computer (Rayner et al., 1982). ${ }^{1}$

\section{HARDWARE}

\section{Eye Movement Measurement}

As Figure 1 shows, the EMR records the subject's current eye position with a spatial resolution of $0.17^{\circ}$ of visual angle and with a temporal resolution of $60 \mathrm{~Hz}$. The EMR employs a recording procedure in which an infrared beam is focused on the subject's eye and a detector scans a reflecting spot called an eye mark on the surface of the cornea. The detector consists of a special solid-state image recognition element, which measures 320 pixels horizontally $\times 244$ pixels vertically. The pupil center is measured with a pupil shooting unit (Model V17, NAC Corp.). This unit enables one to determine the center of the pupil with use of an adjustable screw. The difference between the center of the pupil and the corneal reflected location was minimized by this shooting unit. The EMR converts the spot position into $x, y$ coordinates. The output is voltage coded to the current eye position data every vertical retrace or each $17 \mathrm{msec}$. The analog-to-digital (A/D) converter converts the voltage-coded current eye position data into digits in 12-bit resolution and feeds this to the PC's frame buffer. The PC also analyzes the subject's eye movements and reading performance data after the experiment using a videotape recorder. The $x, y$ coordinate data ( $x$ and $y$ positions) are recorded in the videotape recorder video signal blanking period with NAC's proprietary format.

\section{Moving Window Generator}

The A/D converter board is a standard plug-in module for a PC slot. ${ }^{2}$ The data processing unit (either Type 5 or Type 600 from NAC Corp.) is part of the EMR and handles eye movement data as well as videotape recorder information. The text is displayed on a CRT through a moving window generated by the frame buffer. The PC
(Epson PC-286VF; $12 \mathrm{MHz}$ ) converts the current eye position data provided through the A/D converter to a corresponding window position on the screen. The current eye position data are fed into the frame buffer in real time to make the rectangular window move on the CRT screen. ${ }^{3}$ The frame-buffer-generated window can be superimposed on the PC's digital output to the CRT screen, which is superimposed with the text on the analog CRT screen (KD-PC854, 640 $\times 400$ dot resolution, NEC Corp.). The frame buffer driver provides a high-speed window generating manager. The total cost of our PC-based system (excluding the EMR, data processing unit, and videotape recorder) was approximately $\$ 3,700$, which is relatively inexpensive for a moving window generator. ${ }^{4}$

When fixating the text, the subject can see characters within a certain region around the current fixation point as defined by the program. This technique creates a defined window of text for the subject to see at that fixation. When the subject's eyes move, the text in the window area is replaced by an unreadable mask and a new window of text appears at the location of the new fixation. The size of the window may be changed to meet the needs of each experiment.

\section{Data Collection}

The EMR and data processing unit outputs the current eye position data to two different devices: one is the A/D converter, through which the moving window program monitors the subject's current eye position to control the window position in real time, and the other is the videotape recorder. The current eye position data can be collected from either of these devices. The data analysis program is provided for videotape recorder data. It is better to collect data from the videotape recorder than from the moving window program, because data collection requires CPU time and therefore can cause a substantial delay in control of the moving window if the PC is not fast enough to do both tasks quickly.

\section{SOFTWARE AND OPERATION FLOW}

\section{Flow of the Moving Window Generator}

Under program control, the frame buffer masks the PC's generic screen with the frame buffer's blank screen. The other program displays reading material on the PC screen and passes control to the moving window program. The moving window program signals the frame buffer to unmask a certain area in a certain location of the screen.

Table 1

Time Lag (in Milliseconds) Between Eye and Window Movement

\begin{tabular}{lcccc}
\hline Time & $\begin{array}{c}\text { Eye Mark } \\
\text { Recorder }\end{array}$ & $\begin{array}{c}\text { Moving } \\
\text { Window }\end{array}$ & $\begin{array}{c}\text { CRT } \\
\text { Refresh }\end{array}$ & Total \\
\hline Min & 0 & 1.8 & 0 & 1.8 \\
Max & 33 & 1.8 & 17 & 51.8 \\
Average & 16.5 & 1.8 & 8.5 & 26.8 \\
\hline
\end{tabular}

Note-Eye mark recorder Type 5 (NAC Corporation). 
The unmasked area follows the subject's current eye position as a moving window. To specify the region of the window, the frame buffer's window function uses 5 bytes of data. The moving window program waits for refreshed current eye position data from the A/D converter and calculates the 5 bytes on the basis of the experimental condition and the subject's eye position. The moving window program then waits for the vertical retrace period of the stimulus display CRT and outputs the current eye position data through the $\mathrm{I} / \mathrm{O}$ ports of the converter board. Consequently, it takes only $1.8 \mathrm{msec}$ to specify a single window. This is well within one vertical retrace period of the CRT raster. Table 1 shows the time lag between eye movement and window movement. The time lags in the EMR, moving window, and CRT are also shown in Table 1. These vary from 1.8 to $51.8 \mathrm{msec}$ (average, $26.8 \mathrm{msec}$ ), depending on the sampling and CRT refresh timing. An operational flow chart for the moving window program is shown in Figure 2.

As Figure 2 indicates, the size of the window and the relative fixation position can be defined (in pixels) prior to the moving window session. The window usually generates a rectangular aperture with a defined relative fixation

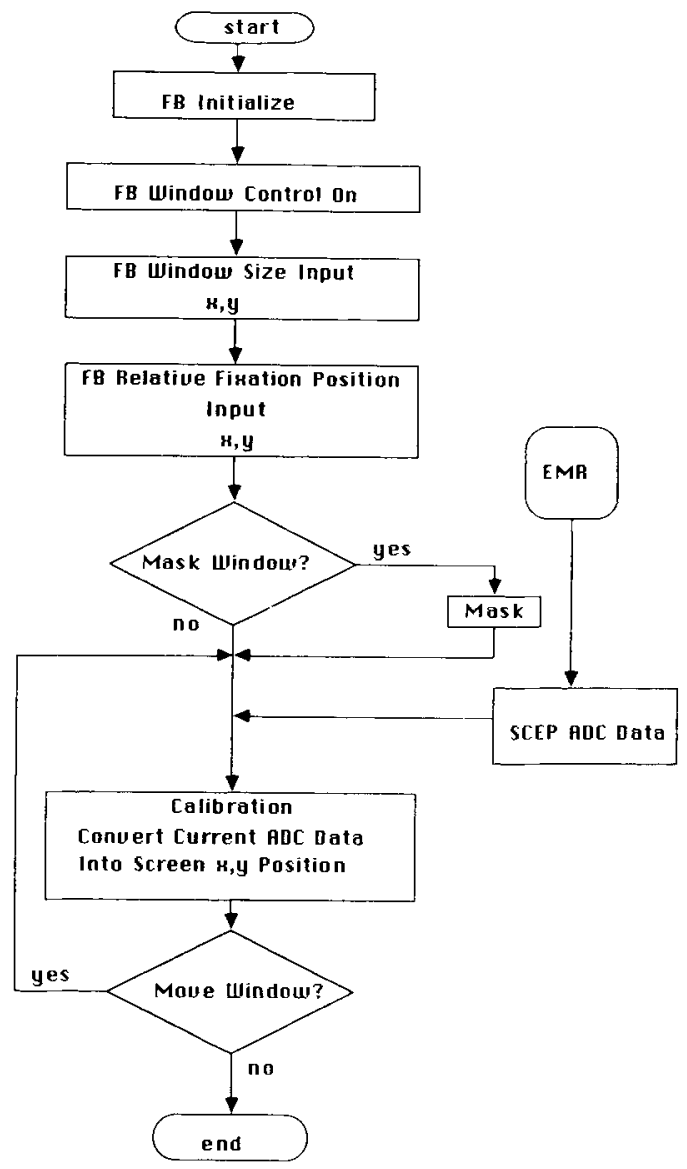

Figure 2. Flow chart of the moving window generator system. FB, frame buffer; EMR, eye movement recorder; current eye position is that of subject.

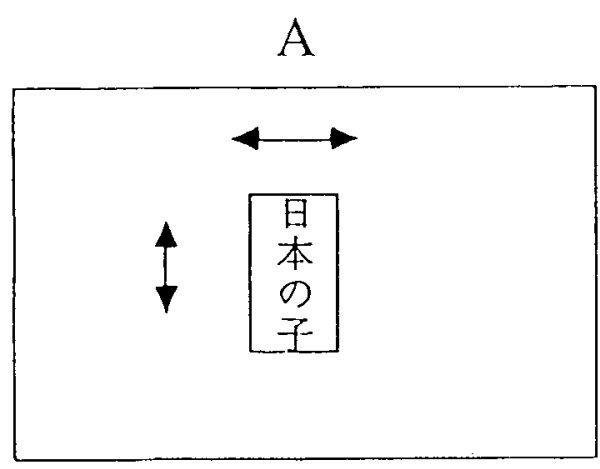

$\mathrm{B}$

\begin{tabular}{|c|c|c|c|c|c|c|}
\hline$\sigma$ & 英 & 自 & $\grave{b}$ & 名 & $と$ & 办 \\
\hline 漠 & 語 & 由 & & 文 & $\notin$ & $\omega$ \\
\hline 字 & 国 & に & & 字 & & $j$ \\
\hline t & 民 & 表 & & \pm & 五 & $\hat{t}$ \\
\hline 知 & だ & 記 & & 元 & 士 & $\sigma$ \\
\hline$\dot{b}$ & と & す & & 覚 & 音 & が \\
\hline な & 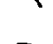 & る & 供 & え & $\bar{\sigma}$ & あ \\
\hline$<$ & $\Sigma$ & $=$ & は & れ & ほ & る \\
\hline
\end{tabular}

Figure 3. (A) Example of a vertical moving window with window size of four characters. (B) Example of a vertical masking window of the same size.

position through which the text is observed. The surrounding background is occupied by a dark masking field (Figure $3 \mathrm{~A}$ ). However, the window can also be changed to a masking field (Figure 3B), in which the subject can observe text with peripheral vision only.

Text

The text for each reading experiment can be generated with a word processor and stored on the hard disk in a text format. Green characters $(x=0.315, y=0.600$, CIE chromaticity values) are displayed on a dark background. One Japanese character requires 2 bytes. Since the text can be treated as graphic data, any text can be displayed in a horizontal or vertical direction with a variable interline, intercolumn, and intercharacter space in pixels.

The main program for the window generator was written in Microsoft C (Version 5.1) at source level and works with MS-DOS (Version 3.3).

\section{TESTING THE SYSTEM}

\section{Noise Problems in Eye Movement Data}

Eye mark recording has advantages in noise reduction over electro-oculogram methods. Muscular activities and external noise do not interfere with the bioelectrical 
change generated by the eye movements. Moreover, the current eye position data are handled digitally, which reduces the amount of noise. However, there are two possible sources of noise. First, when the subject closes his/her eyes, the EMR outputs no data for a moment. Second, when the subject's current eye position is at the edge of two adjacent resolvable locations, the EMR output fluctuates between the two locations. The moving window program does not have any noise reduction mechanism. It simply generates the window following EMR output. When a subject's eyelids are voluntarily shut, the window will move to the system's null position, at the upper left corner of the screen. As soon as the subject opens his/her eyes, the window will recover its previous location (or a new one if the subject has changed fixation).

The measured delay time between saccade initiation and window movement is $26.8 \mathrm{msec}$ on the average. ${ }^{5}$ This lag appears to place some constraints on the type of research that can be explored (see McConkie et al., 1978). The adequacy of this time resolution depends on the aspects of reading or perceptual processes being evaluated. However, the system appears to be adequate for measuring the approximate size of the effective visual field during attention-demanding Japanese text reading in which the saccade size as well as velocity are relatively small in comparison with those of English text reading (Osaka, 1989, 1992).

\section{PRELIMINARY DATA COLLECTION}

\section{Purpose}

The data were collected only for an initial evaluation. This minimal amount was sufficient for calibration and evaluation.

\section{Subjects}

Four college students served as subjects.

\section{Materials}

Five different window sizes, with $1,2,4,8$, or 12 characters, were used during vertical reading of Japanese text. ${ }^{6}$. The vertical window length changed, but a fixed width of 2 characters (20 pixels) was maintained. The current fixation position defined the center of each window. The separation distance between character columns was maintained at 30 pixels, and the vertical intercharacter distance was maintained at 1 pixel.

The text, 16 columns $\times 22$ characters, subtended a $17^{\circ} \times 20^{\circ}$ visual angle and a single character subtended a visual angle of $0.78^{\circ}$ from a $40-\mathrm{cm}$ viewing distance.

\section{Calibration}

Eye movement calibration was conducted before each experiment with each subject. Eight locations were used for initial calibration: the four corner locations of the screen, plus four more locations, each one halfway between adjacent corners. Initial calibration was done in such a way that the measuring area converted to the EMR's entire output range. This ensured that the generated moving window covered the entire measuring area. The current eye position data were calibrated to coincide with the defined fixation position.

\section{Procedure}

After the initial calibration, the subject was asked to read the text silently and naturally from the top down as it appeared on the screen. Thirty saccades were obtained for each window size. Fixation within an area of $1^{\circ}$ of spatial extent and longer than $66 \mathrm{msec}$ was selected as the fixation criterion (the criterion could be changed during data analysis, when the eye movement statistical analysis package was used). Saccade length was measured, but regressive movements due to return sweeps (less than 1\%) were excluded. Each subject had two sessions in each of five window conditions.

\section{Evaluation Trial}

The area of the visual field from which useful information can be extracted during reading is called the effective visual field for reading. Effective visual field size for Japanese text reading in this test was approximately 5-6 characters. This was estimated in terms of vertical saccade length measurements as a function of window size across 4 subjects, with an average of 30 saccades for each subject. The magnitude of saccade was evaluated in terms of character length (Morrison \& Rayner, 1981). The magnitude of saccade was independent of the window size when sizes were between 8 and 12 . However, it tended to decrease as the window size decreased to 4 . Critical window size was measured in terms of change in saccade length. These results concur with those of Osaka (1990), who did not use a moving window, and Osaka and Oda (1991), who did use a moving window.

When foveal vision, corresponding to 3-5 character spaces centered on the current fixation point, was masked (mask window) in the present session, reading Japanese text was almost impossible. This is also true for the reading of English text when reading rate is used as an index, although the size of the critical window increases up to some 11-17 characters from the fixation point (Rayner \& Bertera, 1979).

\section{SUMMARY}

Although the measured lag between the initiation of eye movement and the concomitant window movement appears to place some constraints on the type of research in which the present system can be used, this system is likely to be applicable in a wide range of experimental settings, including studies of reading. The adequacy of its time resolution depends on the aspects of reading or perceptual processing being evaluated. Note that several modifications are needed when one is using some IBMPC-compatible systems, because of a slot feature difference (see notes 2 and 3 for more detail).

Availability. The source code for the moving window generator and the frame buffer window controlling program can be obtained by sending one 2DD-formatted 5-in. floppy disk to the first author. The software also is available by electronic mail by request to the first author at b52046@tansei.cc.u-tokyo.ac.jp. MS-DOS 3.3 or higher and Microsoft $C$ are required. Since the Epson PC286VF, running under MS-DOS (Version 3.3), is only partly compatible with an IBM PC or its clones, minor modifications in address mapping of the moving window program will be needed. Other types of eye movement recording apparatus with analog output could be substituted.

\section{REFERENCES}

BOUma, H., \& DE VoOGD, A. H. (1974). On the control of eye saccades in reading. Vision Research, 14, 273-284.

FEINBERG, R. (1949). A study of some aspects of peripheral visual acuity. American Journal of Optometry, 26, 49-56. 
Legge, G. E., Pelli, D. G., Rubin, G. S., \& SChleske, M. M. (1985) Psychophysics of reading: I. Normal vision. Vision Research, 25, 239-252.

MARCEL, T. (1974). The effective visual field and the use of the context in fast and slow readers of two ages. British Journal of Psychology, 65, 479-492.

McConkie, G. W., \& Rayner, K. (1975). The span of the effective stimulus during a fixation in reading. Perception \& Psychophysics, 17, $578-586$.

McConkie, G. W., Zola, D., Wolverton, G. S., \& Burns, D. D (1978). Eye movement contingent display control in studying reading. Behavior Research Methods \& Instrumentation, 10, 154-166.

Morrison, R. E., \& Rayner, K. (1981). Saccade size in reading depends upon character spaces and not visual angle. Perception \& Psychophysics, 30, 395-396.

Newman, E. B. (1966). The speed of reading when the span of letters is restricted. American Journal of Psychology, 79, 272-278.

OsaKa, N. (1989). Eye fixation and saccade during kana and kanji text reading: Comparison of English and Japanese text processing. Bulletin of the Psychonomic Society, 27, 548-550.

OsaKA, N. (1990). Spread of visual attention during fixation while reading Japanese text. In R. Groner, G. d'Ydewalle, \& R. Parham (Eds.), From eye to mind: Information acquisition in perception, search, and reading (pp. 167-178). Amsterdam: Elsevier.

OSAKA, N. (1992). Size of saccade and fixation duration of eye movements during reading: Psychophysics of Japanese text processing. Journal of the Optical Society of America A, 9, 5-13.

OsaKA, N., \& ODA, K. (1991). Effective visual field size necessary for vertical reading during Japanese text processing. Bulletin of the Psychonomic Society, 29, 345-347.

Poulton, E. C. (1962). Peripheral vision, refractoriness and eye movements in fast oral reading. British Journal of Psychology, 53, 409-419.

RAYNer, K., \& Bertera, J. H. (1979). Reading without a fovea. Science, 206, 468-469.

Rayner, K., \& Pollatsek, A. (1989). The psychology of reading. Englewood Cliffs, NJ: Prentice-Hall.

Rayner, K., Well, A. D., Pollatsek, A., \& Bertera, J. H. (1982). The availability of useful information to the right of fixation in reading. Perception \& Psychophysics, 31, 537-550.

YounG, L. R., \& SHEENA, D. (1975). Survey of eye movement recording methods. Behavior Research Methods \& Instrumentation, 7, 397-429.

\section{NOTES}

1. In McConkie and Rayner's (1975) experiment, two factors, the filter and sampling rate, resulted in a variable lag that could be as long as $40 \mathrm{msec}$ at times. In Rayner et al.'s (1982) experiment, the signal from the eyetracker was sampled every $1 \mathrm{msec}$, and the position of the eye was determined every $4 \mathrm{msec}$. In addition, the display changes associated with each eye movement were accomplished within $5 \mathrm{msec}$ of the completion of the saccade.

2. The A/D converter board (Analog-Pro) is controlled by $A / D$ converter handling software written in Microsoft $\mathrm{C}$ and running on MS-DOS. This commonly used 12 -bit converter board is fully compatible with NEC's PC9801VX, but appears not to be compatible with the IBM PCs due to the difference in slot size. Minor modifications in address mapping of the moving window program are required when one is using an IBM-PC or compatible with an appropriate plug-in $\mathrm{A} / \mathrm{D}$ converter board.

3. The frame buffer (HyperFrame) board is controlled by a utility program, including a handling library, written in Microsoft $\mathrm{C}$. It has a $768 \mathrm{~K}$ video memory so that $640 \times 400$ pixels on a CRT screen can be covered. The analog output (400 lines vertically with horizontal synchronization frequency of $24.83 \mathrm{kHz}$ ) should be connected to an analog CRT. This board is also a commonly used video memory board, fully compatible with NEC's PC9801VX. It appears not to be compatible with IBM PCs, owing to their slot size difference. Minor modifications in address mapping of the moving window program are required when one is using an IBM PC or compatible with an appropriate plug-in frame buffer board.

4. The A/D converter board, with user's manual, costs about $\$ 1,400$ (Canopus Corp.: 1-4-30 Nishiokamoto, Higashinada-ku, Kobe 658, Japan). The frame buffer board (HyperFrame), with handler disk and user's manual, costs about $\$ 750$ (Digital Arts Corp.: Shinsei bldg 5F, 2-3-7 Shibuya, Shibuya-ku, Tokyo 150, Japan). The PC system costs about $\$ 1,550$.

5. Replacing the EMR-5 (present system) with the EMR-600 (recently announced version) results in a shorter lag between eye movement and window movement (average of $6+1.8+8.5=16.3 \mathrm{msec}$ with minimal and maximal of 1.8 and $24.8 \mathrm{msec}$, respectively; the moving window software and CRT refresh requires 1.8 and $8.5 \mathrm{msec}$, respectively; see Table 1) without changing the main system requirements. Both types of EMR incorporate a solid-state image precognition element. A corneal reflection method is employed for measuring eye movements.

6 . Vertical text was used in this study, because previous studies showed no critical difference between vertical and horizontal reading during Japanese text processing (Osaka, 1989, 1990). The average kanji contribution factor (kanji contribution factor = [number of kanji characters]/ [number of characters in whole text]) in the present texts (averaged across 18 texts) was $0.26(S D=0.05)$. Punctuation marks contributed an average of $0.07(S D=0.006)$. Hirakana and katakana were assumed to be equivalent. Kanji familiarity was controlled by using standard high school texts. There is no significant difference in readability of text in the range between 0.2 and 0.4 , the kanji contribution factor.

(Manuscript received February 27, 1991;

revision accepted for publication July 9,1993 .) 